\title{
High-order effective-medium theory of subwavelength gratings in classical mounting: application to volume holograms
}

\author{
Philippe Lalanne and Jean-Paul Hugonin \\ Institut d'Optique Théorique et Appliquée, Centre National de la Recherche Scientifique, BP 147, \\ F 91403 Orsay Cedex, France
}

Received December 1, 1997; revised manuscript received February 19, 1998; accepted March 2, 1998

\begin{abstract}
We derive closed-form expressions for the effective index of subwavelength gratings up to the fourth and the second order for TE and TM polarization, respectively. These expressions are valid for arbitrary grating structures and are a generalization of previous results obtained for lamellar gratings with one groove per period (a structure often called a two-component layered medium). The effective-medium-theory predictions are carefully validated with exact electromagnetic theories for slanted and unslanted sinusoidally modulated volume gratings and for classical mounting. It is shown that, even for large period-to-wavelength ratios near the cutoff value, the form birefringence is accurately predicted at any angle of incidence. (C) 1998 Optical Society of America [S0740-3232(98)01107-7]
\end{abstract}

OCIS codes: $050.1970,050.7330,260.1440$.

\section{INTRODUCTION}

Homogenization theories have been applied to a large variety of fields, covering mechanics, acoustics, physics, mathematics, and chemistry, for example. The objective of homogenization is to provide a simplified model of composite materials whose rigorous analysis is computationally difficult and sometimes even impossible. In general, homogenization theories exploit the fact that two different scales are relevant for studying the material properties. On a microscopic scale, the main characteristic of the composite material is heterogeneity. Conversely, on the scale of the observation, the microscopic structure is indistinguishable, and the material appears homogeneous. Homogenization theory or effective-medium theory (EMT) exploits this dual scale by introducing a small parameter $\chi$ that is defined by the ratio of two characteristic lengths associated with the two scales. When $\chi$ tends to zero (asymptotic limit or static limit), the properties of the material and of its homogenized version are identical. Interesting results for engineers are obtained when simple approximate expressions are available for the nonasymptotic case.

Hereafter we focus on the homogenization of onedimensional (1-D) subwavelength gratings. A subwavelength grating is defined as a periodic structure, incorporated between two semi-infinite media, that does not diffract light in the far field (only the zeroth reflected and transmitted orders are propagating for a given incident plane wave). Recent experimental and theoretical investigations have shown that periodic subwavelengthstructured surfaces with periods that are small compared with the illumination wavelength behave as homogeneous media. The investigations have also suggested interesting applications, such as fabrication of antireflection coatings, ${ }^{1-6}$ quarter-wave plates, ${ }^{7,8}$ polarizers, ${ }^{9}$ and graded-phase diffractive elements, ${ }^{10,11}$ for example. The small parameter $\chi$ used for the homogenization problem is the period-to-wavelength ratio $(\Lambda / \lambda)$, and the effective index $n_{\text {eff }}$ is seen as a power series of $\Lambda / \lambda$ :

$$
n_{\text {eff }}=n_{0}+n_{1} \Lambda / \lambda+n_{2}(\Lambda / \lambda)^{2}+\ldots
$$

Recently it was shown ${ }^{12}$ that the power series of Eq. (1) does not provide a complete description of the homogenization problem; for an adequate homogenized model, another parameter $\chi$ equal to the depth-to-wavelength ratio must also be considered, in addition to $\Lambda / \lambda$, to take into account evanescent modes. The impact of evanescent modes on the effective properties of the grating is especially stringent for grating depths smaller than a quarter wave (the birefringence $\Delta n$ of $1-\mathrm{D}$ and two-dimensional gratings vanish for small depth-to-wavelength ratios). In the following we assume that the grating depth is large enough so that evanescent modes can be neglected. This amounts to considering that the effective index of the grating in Eq. (1) is given by the effective index of the fundamental propagating mode supported by the periodic structure.

The properties of 1-D periodic structures have been analyzed in great detail. Two different kinds of work can be distinguished. On the one hand, theoreticians have made drastic efforts to prove the equivalence of 1-D gratings and homogeneous uniaxial thin films in the static limit (see, for instance, Refs. 13 and 14). On the other hand, other studies have focused on the derivation of closed-form expressions for the effective index in the nonstatic limit. Closed-form expressions up to order 4 for TE and TM polarizations are available for 1-D periodic lamellar gratings composed of two homogeneous materials (a structure often called two-layered media); see, for instance, Refs. 6, 15, and 16. The homogenization of twocomponent layered media is made easy by the fact that the permittivity is piecewise constant and thus by the fact 
that the modes supported by the structure are analytically known. By using a Fourier expansion of the field inside the periodic structure, Bell et al. ${ }^{17}$ derived closedform expressions for the second-order effective index of 1-D symmetric gratings and for TE polarization. More recently, Lalanne and Lemercier-Lalanne ${ }^{18}$ obtained closed-form expressions up to order 2 for TE and TM polarizations of arbitrary (symmetric or not) 1-D gratings but restricted their analysis to gratings illuminated under normal incidence.

In this paper we derive closed-form expressions for the effective index of arbitrary gratings in classical mounting. For TE polarization, the expression includes terms up to order 4. For TM polarization, it includes terms up to order 2 . The methodology used for the derivation is presented in Subsection 2.A, and the main formulas are summarized in Subsection 2.B. The case of practical interest of volume gratings is considered in Sections 3 and 4 for unslanted and slanted gratings, respectively. By comparison with exact electromagnetic theories, it is found that the birefringence is accurately predicted by EMT up to period-to-wavelength ratios equal to 0.7 times the cutoff value.

\section{EFFECTIVE-MEDIUM THEORY}

The 1-D grating diffraction problem considered in the following analysis is depicted in Fig. 1. A linearly polarized electromagnetic plane wave is obliquely incident at an ar-

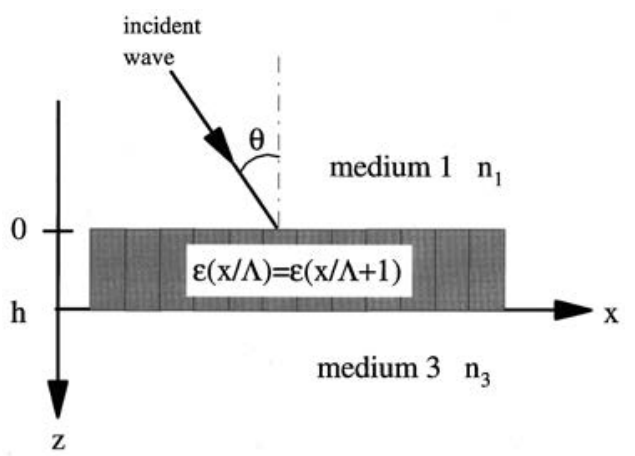

(a)

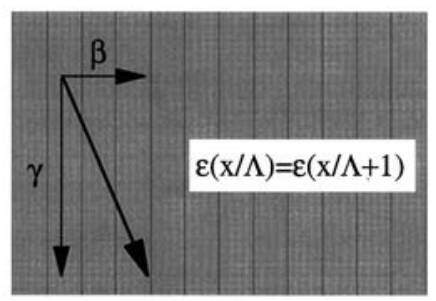

(b)

Fig. 1. (a) Geometry for the nonconical grating diffraction problem analyzed in the paper. The relative permittivity is assumed to be independent of the $z$ direction, and an unslanted grating is considered in the figure ( $\epsilon$ depends only on the $x$ coordinate). (b) Corresponding periodic structure with an infinite spatial extent in the $z$ direction. $\quad \beta$ and $\gamma$ are the normalized $x$ and $z$ components of the wave vector along the $x$ and the $z$ direction, respectively. bitrary angle of incidence $\theta$ on a dielectric or a lossy grating in a classical mounting (the plane of incidence is perpendicular to the $y$ direction). The wavelength in the vacuum of the incident wave is denoted by $\lambda$, and the corresponding wave-vector length $k$ is equal to $2 \pi / \lambda$. The grating region is composed of a $1-D$ periodic structure along the $x$ axis. The grating period is denoted by $\Lambda$ and the relative permittivity by $\epsilon(x / \Lambda)$. The grating is surrounded by two different media with refractive indices $n_{1}$ and $n_{3}$. The $z$ axis is perpendicular to the grating boundaries, and the diffraction problem is invariant in the $y$ direction. A temporal dependence of $\exp (-j \omega t)$ of the incident wave is assumed $\left(j^{2}=-1\right)$ throughout the paper. Magnetic effects are not considered. We denote by $\epsilon_{n}$ and $a_{n}$ the $n$th Fourier coefficients of $\epsilon$ and $\epsilon^{-1}$, respectively.

\section{A. Methodology}

As is mentioned in Section 1, we consider a periodic structure with a relative permittivity identical to that of the grating but with an infinite spatial extent in the $z$ direction [see Fig. 1(b)]. This amounts to neglecting the effect of evanescent waves in the diffraction problem of Fig. 1(a) and considering only the fundamental mode supported by the periodic structure. For TE polarization the interesting field quantity is the $y$ component of the electric field. The Helmoltz equation is

$$
\frac{\partial^{2} E_{y}}{\partial z^{2}}+\frac{\partial^{2} E_{y}}{\partial x^{2}}+\epsilon k^{2} E_{y}=0 .
$$

Similarly, for TM polarization, we have

$$
\frac{\partial^{2} H_{y}}{\partial z^{2}}+\epsilon \frac{\partial}{\partial x}\left(\frac{1}{\epsilon} \frac{\partial H_{y}}{\partial x}\right)+\epsilon k^{2} H_{y}=0,
$$

where $H_{y}$ is the $y$ component of the magnetic field. With a dimensionless parameter $\alpha$ ( $\alpha=0$ and 1 for TE and TM polarizations, respectively), Eqs. (1) and (2) can be rewritten as

$$
\frac{\partial^{2} U}{\partial z^{2}}+\epsilon^{\alpha} \frac{\partial}{\partial x}\left(\frac{1}{\epsilon^{\alpha}} \frac{\partial U}{\partial x}\right)+\epsilon k^{2} U=0,
$$

where $U$ is a function of $x$ and $z$. Because $\epsilon$ does not depend on $z$, we look for a solution of the form

$$
U(x, z)=\exp (j k \gamma z) V_{1}(x / \Lambda) .
$$

Incorporating the expression of $U$ into Eq. (4) and denoting by $V_{2}$ the quantity

$$
\frac{1}{\epsilon^{\alpha}} \frac{\lambda}{2 \pi \Lambda} \frac{\mathrm{d} V_{1}}{\mathrm{~d} x^{\prime}}
$$

where $x^{\prime}=x / \Lambda$, we obtain

$$
\epsilon^{\alpha} \frac{\mathrm{d} V_{2}}{\mathrm{~d} x^{\prime}}+\frac{2 \pi \Lambda}{\lambda}\left(\epsilon-\gamma^{2}\right) V_{1}=0 .
$$

Equation (6) can be written as

$$
\frac{\mathrm{d}}{\mathrm{d} x^{\prime}}\left(\begin{array}{l}
V_{1} \\
V_{2}
\end{array}\right)=\frac{2 \pi \Lambda}{\lambda}\left[\begin{array}{cc}
0 & a\left(x^{\prime}\right) \\
b\left(x^{\prime}\right) & 0
\end{array}\right]\left(\begin{array}{l}
V_{1} \\
V_{2}
\end{array}\right)
$$

or, equivalently, as 


$$
\frac{\mathrm{d}}{\mathrm{d} x^{\prime}} \mathbf{V}=\frac{2 \pi \Lambda}{\lambda} \mathbf{M V}
$$

In Eq. (8), $\mathbf{V}$ denotes the vector formed by coefficients $V_{1}$ and $V_{2}$, and $\mathbf{M}$ is the $2 \times 2$ matrix on the right-hand side of Eq. (7). The functions $a\left(x^{\prime}\right)$ and $b\left(x^{\prime}\right)$ are defined by $a\left(x^{\prime}\right)=\epsilon^{\alpha}$ and $b\left(x^{\prime}\right)=\left(\gamma^{2}-\epsilon\right) / \epsilon^{\alpha}$. Equation (8) is a first-order linear differential equation with nonconstant coefficients. For a given value of $\beta$ we can look for a solution of the form $\mathbf{V}=\exp \left(j k \beta x^{\prime} \Lambda\right) \mathbf{v}\left(x^{\prime}\right)$ without loss of generality. The related differential equation in $\mathbf{v}$ is

$$
\frac{\mathrm{d}}{\mathrm{d} x^{\prime}} \mathbf{v}=\frac{2 \pi \Lambda}{\lambda}(\mathbf{M}-j \beta \mathbf{I}) \mathbf{v},
$$

where $\mathbf{I}$ is the identity matrix. The general solution of Eq. (9) can be written as $\mathbf{v}\left(x^{\prime}\right)=\mathbf{C}\left(x^{\prime}\right) \mathbf{v}(0)$, where $\mathbf{C}\left(x^{\prime}\right)$ is a $2 \times 2$ matrix whose coefficients can be computed by numerical integration. As explained below, we are concerned in the following with the matrix $\mathbf{C}(1)$, which we simply denote by $\mathbf{C}$. If we assume that $(2 \pi \Lambda) / \lambda \ll 1$, it is easy to determine that an approximate expression for $\mathbf{C}$ is

$$
\begin{aligned}
\mathbf{C}=\mathbf{I} & +\frac{2 \pi \Lambda}{\lambda} \mathbf{C}_{1}(1)+\left(\frac{2 \pi \Lambda}{\lambda}\right)^{2} \mathbf{C}_{2}(1) \\
& +\left(\frac{2 \pi \Lambda}{\lambda}\right)^{3} \mathbf{C}_{3}(1)+O\left(\frac{2 \pi \Lambda}{\lambda}\right)^{4}
\end{aligned}
$$

where $\quad \mathbf{C}_{1}\left(x^{\prime}\right)=\int_{0}^{x^{\prime}}(\mathbf{M}-j \beta \mathbf{I}) \mathrm{d} t, \quad \mathbf{C}_{2}\left(x^{\prime}\right)=\int_{0}{ }^{x^{\prime}}(\mathbf{M}$ $-j \beta \mathbf{I}) \mathbf{C}_{1} \mathrm{~d} t$ and $\mathbf{C}_{3}\left(x^{\prime}\right)=\int_{0}^{x^{\prime}}(\mathbf{M}-j \beta \mathbf{I}) \mathbf{C}_{2} \mathrm{~d} t$. For the series expansion of Eq. (10) to be valid, the integrals of $\epsilon$ and $\epsilon^{-1}$ have to be defined over the interval $[0 ; 1]$, which is guaranteed for physical reasons. For $(k \beta)$ to be the $x$ component of the propagating wave vector, a vector $\mathbf{v}^{*}$ verifying $\mathbf{v}^{*}(1)=\mathbf{C v}^{*}(0)$ and $\mathbf{v}^{*}(1)=\mathbf{v}^{*}(0)$ has to exist (pseudoperiodicity condition). Here $\mathbf{v}^{*}$ is an eigenvector of matrix $\mathbf{C}$, and the corresponding eigenvalue is equal to one. In other words, the determinant of matrix $\mathbf{C}-\mathbf{I}$ is equal to zero, and the dispersion relation, up to the second order of $\Lambda / \lambda$, can be written as

$$
\begin{aligned}
\| \mathbf{C}_{1}(1)+(2 \pi \Lambda / \lambda) \mathbf{C}_{2}(1)+(2 \pi \Lambda / \lambda)^{2} \mathbf{C}_{3}(1) & \\
& +O(2 \pi \Lambda / \lambda)^{3} \|=0 .
\end{aligned}
$$

\section{B. General Formula}

For reasons of brevity we now omit the long but elementary intervening calculations and give only the results. We denote by $a^{*}\left(x^{\prime}\right), b^{*}\left(x^{\prime}\right), A\left(x^{\prime}\right)$, and $B\left(x^{\prime}\right)$, the quantities defined by

$$
\begin{aligned}
a^{*}\left(x^{\prime}\right) & =a\left(x^{\prime}\right)-\epsilon_{0}{ }^{\alpha}, \\
b^{*}\left(x^{\prime}\right) & =b\left(x^{\prime}\right)-\gamma^{2} a_{0}{ }^{\alpha}+\epsilon_{0}^{1-\alpha}, \\
A\left(x^{\prime}\right) & =\int_{0}^{x^{\prime}} a^{*}(x) \mathrm{d} x, \\
B\left(x^{\prime}\right) & =\int_{0}^{x^{\prime}} b^{*}(x) \mathrm{d} x,
\end{aligned}
$$

respectively. By keeping all terms up to the second order of $\Lambda / \lambda$ in Eq. (11), we obtain the following dispersion relation:

$$
\epsilon_{0}^{\alpha}\left(\gamma^{2} a_{0}^{\alpha}-\epsilon_{0}^{1-\alpha}\right)+\beta^{2}=(2 \pi \Lambda / \lambda)^{2} R
$$

with

$$
\begin{aligned}
R= & \epsilon_{0}{ }^{2 \alpha}\langle B \mid B\rangle+\frac{\beta^{4}}{\epsilon_{0}{ }^{2 \alpha}}\langle A \mid A\rangle+2 \beta^{2}\langle B \mid A\rangle \\
& -2 \epsilon_{0}{ }^{\alpha}\left\langle B \mid b^{*} A\right\rangle+2 \frac{\beta^{2}}{\epsilon_{0}{ }^{\alpha}}\left\langle A \mid a^{*} B\right\rangle-\left\langle a^{*} \mid B\right\rangle^{2} .
\end{aligned}
$$

In Eq. (12b), the covariance $\langle f \mid g\rangle$ of two functions $f$ and $g$ is defined by $\langle f \mid g\rangle=\int_{0}^{1}\left[f(x)-\int_{0}^{1} f\left(x^{\prime}\right) \mathrm{d} x^{\prime}\right][g(x)$ $\left.-\int_{0}^{1} g\left(x^{\prime}\right) \mathrm{d} x^{\prime}\right] \mathrm{d} x$. Basically, the computation of products $\langle f \mid g\rangle$ in Eq. (12b) relies on integrals of functions $\epsilon$ and $\epsilon^{-1}$ and on products of these functions. Equations (12a) and (12b) are the main practical results of this paper. They are valid for TE and TM polarizations (up to the second order of $\Lambda / \lambda$ ) and for any kind of periodic structures, as long as the permittivity depends only on the $x$ coordinate. They hold for the two practically important cases of unslanted lamellar and volume gratings. The case of slanted grating is discussed in Section 4 .

In general, one is interested in knowing the effective in$\operatorname{dex} n_{\text {eff }}$ of the subwavelength grating for a given angle of incidence $\theta$. This amounts to imposing the $x$ component of the normalized propagating wave vector $\beta$ by the relationship

$$
\beta=n_{1} \sin (\theta) .
$$

In Eq. (12b), $R$ is a function of both $\beta$ and $\gamma$. We transform it into a function depending only on $\beta$ by replacing $\gamma$ with its zeroth-order approximate expression $\gamma^{2}=\left(\epsilon_{0}\right.$ $\left.-\beta^{2}\right) /\left(\epsilon_{0}{ }^{\alpha} a_{0}{ }^{\alpha}\right)$ in the expression of $R$. Thus the effective relative permittivity $\epsilon_{\text {eff }}\left(\epsilon_{\text {eff }}=n_{\text {eff }}^{2}\right)$ is given by

$$
\epsilon_{\mathrm{eff}}=\beta^{2}+\gamma^{2},
$$

with

$$
\gamma^{2}=f(\beta)=\frac{1}{\epsilon_{0}{ }^{\alpha} a_{0}{ }^{\alpha}}\left[\epsilon_{0}-\beta^{2}+\left(\frac{2 \pi \Lambda}{\lambda}\right)^{2} R(\beta)\right]
$$

For transparent dielectric gratings, $\gamma^{2}$ is either positive or negative. Negative values correspond to total internal reflection at the upper grating interface. In general, for absorbing materials, $\gamma^{2}$ is a complex number.

\section{Remarks}

For TE polarization, $a^{*}$ and $A$ are both equal to zero. The dispersion relation of Eqs. (12a) and (12b) takes a simple form, since only the product $\langle B \mid B\rangle^{2}$ has to be computed. The methodology used above to derive secondorder expressions can be used up to the fourth order without burdensome handwritten computation. For the sake of generality, we now give the effective relative permittivity of the TE wave up to the fourth order of $\Lambda / \lambda$ : 


$$
\begin{aligned}
\epsilon_{\mathrm{eff}}= & \epsilon_{0}+\left(\frac{\Lambda}{\lambda}\right)^{2}\left(\sum_{p \neq 0} \frac{\epsilon_{p} \epsilon_{-p}}{p^{2}}\right)+\left(\frac{\Lambda}{\lambda}\right)^{4} \\
& \times\left(\left(4 \beta^{2}-\epsilon_{0}\right) \sum_{p \neq 0} \frac{\epsilon_{p} \epsilon_{-p}}{p^{4}}+\sum_{p \neq 0, k \neq 0} \frac{\epsilon_{p} \epsilon_{k-p} \epsilon_{-k}}{p^{2} k^{2}}\right) .
\end{aligned}
$$

Equation (16) relies on the Fourier coefficients $\epsilon_{k}$ of the relative permittivity $\epsilon$ rather than on products of type $\langle\cdot \mid \cdot\rangle$. The equation will be easier to use in Section 3 . In general, the fourth-order EMT for TE waves provides accurate results (see Sections 3 and 4 for volume gratings and Section 2 of Ref. 18 for the case of highly modulated lamellar gratings illuminated under normal incidence). From Eq. (16) it is concluded that the normal surface of $\mathrm{TE}$ waves is still a sphere up to the second order; a $\beta$ dependence exists only in the fourth-order term of the effective index. For TM polarization the normal surface is no longer an ellipsoid of revolution up to the second order. and dichromated gelatins. We assume that the relative permittivity in the grating region is $\epsilon(x) / \Lambda$ $=\epsilon_{0}+\Delta \epsilon \cos (2 \pi x / \Lambda)$, where $\epsilon_{0}$ is the bias relative permittivity of the material and $\Delta \epsilon$ is the relative permittivity modulation. To our knowledge only the zeroth-order EMT has been applied to the analysis of such continuously varying permittivity gratings, ${ }^{19}$ and the present paper is the first to present accurate expressions that are valid for large periods near cutoff.

For TE polarization, Eq. (16) becomes

$\mathrm{TE}: \quad \epsilon_{\mathrm{eff}}=\epsilon_{0}+\frac{(\Delta \epsilon)^{2}}{2}\left(\frac{\Lambda}{\lambda}\right)^{2}+2 \beta^{2}(\Delta \epsilon)^{2}\left(\frac{\Lambda}{\lambda}\right)^{4}$.

As mentioned above, a dependence in $\theta$ is observed in the last term of order 4. For TM polarization, the effectiveindex expression is more complex. By including the Fourier coefficients $a_{n}$ of the inverse relative permittivity, we obtain

$$
\mathrm{TM}: \quad \epsilon_{\mathrm{eff}}=\frac{1}{\epsilon_{0} a_{0}}\left(\epsilon_{0}-\beta^{2}+\epsilon_{0} a_{0} \beta^{2}+\left(\frac{\Lambda}{\lambda}\right)^{2}\left\{\begin{array}{l}
\frac{\beta^{4} \Delta \epsilon^{2}}{2 \epsilon_{0}^{2}}+\beta^{2} \Delta \epsilon\left(1-\frac{\beta^{2}}{\epsilon_{0}}\right)\left(\frac{2 a_{1}}{a_{0}}+\frac{\Delta \epsilon a_{2}}{2 \epsilon_{0} a_{0}}\right) \\
+\frac{\epsilon_{0}}{a_{0}^{2}}\left(1+\frac{\beta^{4}}{\epsilon_{0}^{2}}-\frac{2 \beta^{2}}{\epsilon_{0}}\right)\left[\epsilon_{0} \sum_{n \neq 0}\left(\frac{a_{n}}{n}\right)^{2}+2 \Delta \epsilon \sum_{n>0} \frac{a_{n} a_{n+1}}{n(n+1)}\right]
\end{array}\right\}\right) .
$$

Thus the conventional uniaxial crystal approximation for 1-D gratings is strictly valid only in the static limit.

We checked the closed-form expression of Eqs. (12a) and (12b) against results available in the literature:

- For lamellar gratings with one groove per period (two-component layered media), the handwritten computations of products $\langle\cdot \mid \cdot\rangle$ in the expression of $R$ is straightforward, since it requires the computation of integrals of piecewise-constant functions. We found that our EMT results are consistent with those derived by $\operatorname{Rytov}^{15}$ or more recently by $\mathrm{Gu}$ and Yeh. ${ }^{16}$

- For more general grating structures including nonlamellar gratings, such as volume gratings or lamellar gratings with more than one groove per period, the products $\langle\cdot \mid \cdot\rangle$ in Eq. (12b) can be expressed by use of the Fourier coefficients $a_{n}$ and $\epsilon_{n}$. It is easily shown that

* for TE polarization Eqs. (12a) and (12b) are identical to Eq. (15), which was previously derived by Bell et $a l$. in Ref. 17 for grating profiles for which a center of symmetry exists for $\epsilon(x)$;

* for TE and TM polarizations our EMT result is consistent with those previously obtained by Lalanne and Lemercier-Lalanne [see Eqs. (8) and (15) in Ref. 18] for grating structures that are arbitrary but have normal incidence $(\beta=0)$.

\section{UNSLANTED VOLUME GRATINGS}

In this section we are concerned with sinusoidally unslanted modulated volume holograms. These holograms can be recorded into different materials that include, for example, photorefractive crystals, photopolymers, solgels,
For the following numerical tests we assume that the volume hologram is recorded in a dichromated gelatin layer deposited on a glass substrate $\left(n_{3}=1.54\right)$. The incident medium is air $\left(n_{1}=1\right)$, and the grating is $8.8 \mu \mathrm{m}$ thick. The bias permittivity of the hologram is 1.8496 at a wavelength of $0.6328 \mu \mathrm{m}$. This corresponds to a bias index of 1.36. The relative-permittivity modulation $\Delta \epsilon$ is 0.25 , which corresponds to an index modulation of $\sim 0.09$. This experimental situation is identical to that used by Campbell and Kostuk in Ref. 19, in which the limits of validity of zeroth-order EMT for modeling the birefringence properties of sinusoidally modulated volume holograms are analyzed.

We define the cutoff $\kappa$ as the period-to-wavelength ratio $\Lambda_{c} / \lambda$ beyond which the grating of Fig. 1 stops behaving as a zeroth-order filter (some diffracted orders are nonevanescent in the substrate or incident medium). For a given angle of incidence $\theta$, whether a diffraction order propagates is determined by the grating equation. For unslanted gratings, it is found that $\kappa=1 /\left[\max \left(n_{3}, n_{1}\right)\right.$ $+\beta)$, which reduces to $\kappa=1 /\left(n_{3}+\sin \theta\right)$ for the volume hologram considered.

\section{A. Testing Effective-Medium Theory Against Exact Bloch-Wave Computation}

We first present a comparison between the effective-index predictions of Eqs. (17) and (18) and exact values obtained with Bloch-wave computation. ${ }^{20}$ The Bloch-wave method exploits the periodicity of the grating permittivity and relies on a Fourier decomposition of the electromagnetic field quantities. Figure 2 shows the effective relative permittivity of the volume grating as a function of $\beta$ for 
TE polarization. EMT predictions and Bloch-wave computational results correspond to dashed or solid curves and circles, respectively. For period-to-wavelength ratios up to $\kappa / 2$, the agreement between EMT predictions and exact numerical results is excellent: The largest differences equal $2 \times 10^{-4}$ and $3 \times 10^{-5}$ for the second- and fourth-order EMT predictions, respectively. At cutoff the EMT prediction is less accurate, especially for $\beta$ greater than 0.6 (angles of incidence $\theta$ greater than $37^{\circ}$ ). However, it is noticeable that the differences do not exceed 0.005 for the fourth-order EMT approximations and for $\beta$ smaller than $1\left(\theta<90^{\circ}\right)$. Figure 3 shows a comparison for TM polarization. As above, the dashed curves represent the second-order relative effective permittivity [Eq. (18)], and circles are exact values. Once again a good agreement is obtained. It is even better than for TE polarization: The deviation between approximate and exact results does not exceed 0.002 even at cutoff for $\beta$ smaller than 1.

Two interesting features in Figs. 2 and 3 have to be mentioned. First, as $\beta$ increases toward 1.36, the effective relative permittivities found with Bloch-wave computations are nearly identical for TE and TM polarizations. This is easily understood when we consider that, for $\beta \approx 1.36$, waves are propagating along the $x$ direction $(\gamma=0)$, normal to the fringes and are experiencing a thin-film stack (rugate) under normal incidence. Second, it is noteworthy that, for a given value of $\beta$ in Fig. 3 ( $\beta \approx 1$ ), the effective relative permittivity is nearly independent of $\Lambda / \lambda$. This is a general property of periodic structures for which $1 / \epsilon(x)=-p \epsilon(x)+q$, with $p$ and $q$ being two real numbers. These structures include lamellar gratings composed of two materials and also volume

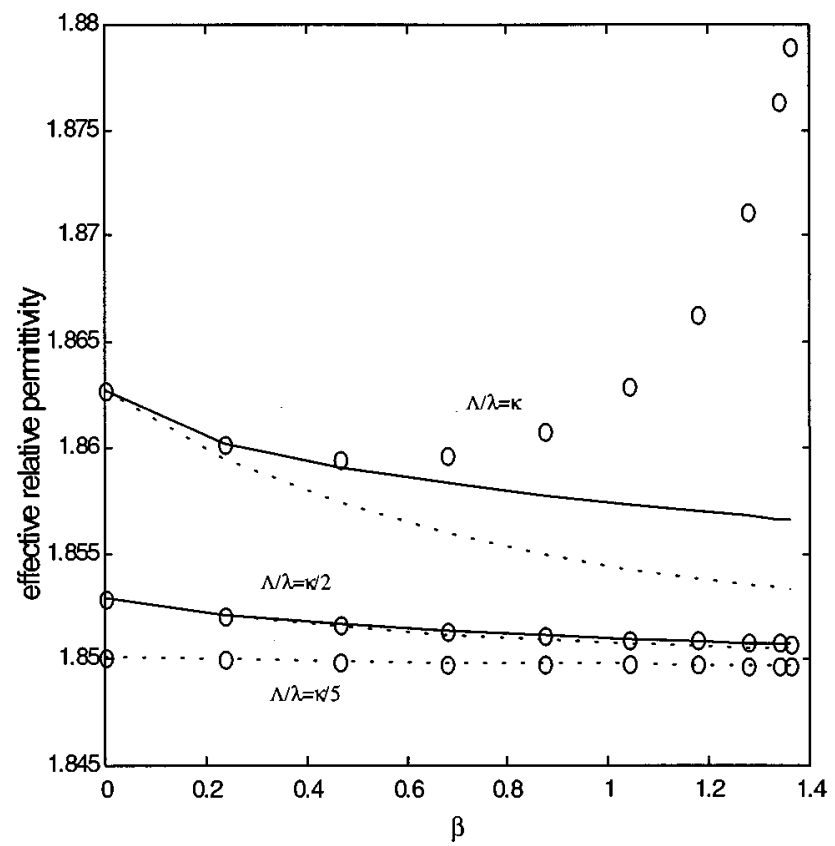

Fig. 2. Effective relative permittivity as a function of the angle of incidence for TE polarization and for different period-towavelength ratios: comparison between EMT predictions (dotted and solid curves) and Bloch-wave computation (circles). Dotted and solid curves are obtained with second-order and fourth-order EMT of Eq. (17), respectively.

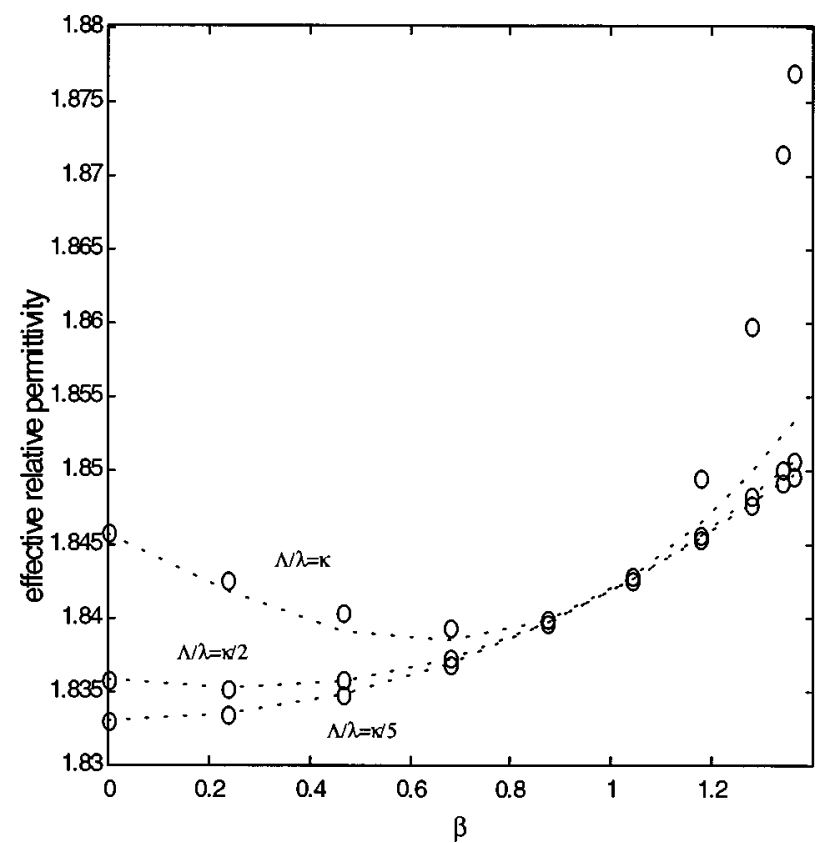

Fig. 3. Effective relative permittivity as a function of the angle of incidence for TM polarization and for different period-towavelength ratios: comparison between the second-order EMT predictions (dotted curves) of Eq. (18) and Bloch-wave computation (circles).

gratings with small permittivity modulations. In fact, it is easily shown that, for these structures, $R(\beta)$ is null for $\beta^{2}=p \epsilon_{0}^{2} \gamma^{2}$. For the volume grating considered in this paper, $\Delta \epsilon \ll \epsilon_{0}$ and $p=1 / \epsilon_{0}{ }^{2}$, because $1 / \epsilon(x)$ $\approx-\epsilon(x) / \epsilon_{0}{ }^{2}+2 / \epsilon_{0}$. Thus $R(\beta)$ is null for $\beta^{2}=\gamma^{2}$. As $\beta^{2}+\gamma^{2}$ is approximately equal to the bias relative permittivity $\epsilon_{0}$, it is predicted that the effective relative permittivity is weakly dependent on $\Lambda / \lambda$ for $\beta$ $=\left(\epsilon_{0} / 2\right)^{1 / 2} \approx 0.96$, as confirmed by Fig. 3 .

\section{B. Testing Effective-Medium Theory Against Exact Diffraction Theory}

The preceding tests do not provide any information about the equivalence (or nonequivalence) of volume gratings and homogeneous thin films. They indicate only that the second- and fourth-order effective indices [Eqs. (17) and (18)] are accurate expressions for the effective relative permittivity of the fundamental mode supported by the sinusoidally modulated structure, even for large period-towavelength ratios near cutoff. In the following we are concerned with testing the equivalence of subwavelength volume holograms and homogeneous thin films. More specifically, we try to answer two questions: (1) In the far field, do the diffraction patterns of subwavelength volume gratings mimic those of homogeneous thin films? and (2) Is the far-field pattern accurately predicted by the second- and fourth-order EMT expressions of Eqs. (17) and (18)? To answer these questions, we note that Fig. 1 defines a diffraction problem that can be solved rigorously. Solving Maxwell's equations and boundary conditions at the grating interfaces, we compute the complex transmitted amplitude $t$ and the reflected amplitude $r$ of the zeroth-order diffracted waves. Then by the analogy between subwavelength gratings and homogeneous thin films, we define the equivalent effective index of the vol- 
ume grating as the real number $n_{\text {RCWA }}$ (where RCWA stands for rigorous coupled-wave analysis) that minimizes the error function

$$
e=\left|r^{\prime}\left(n_{\mathrm{RCWA}}\right)-r\right|+\left|t^{\prime}\left(n_{\mathrm{RCWA}}\right)-t\right|,
$$

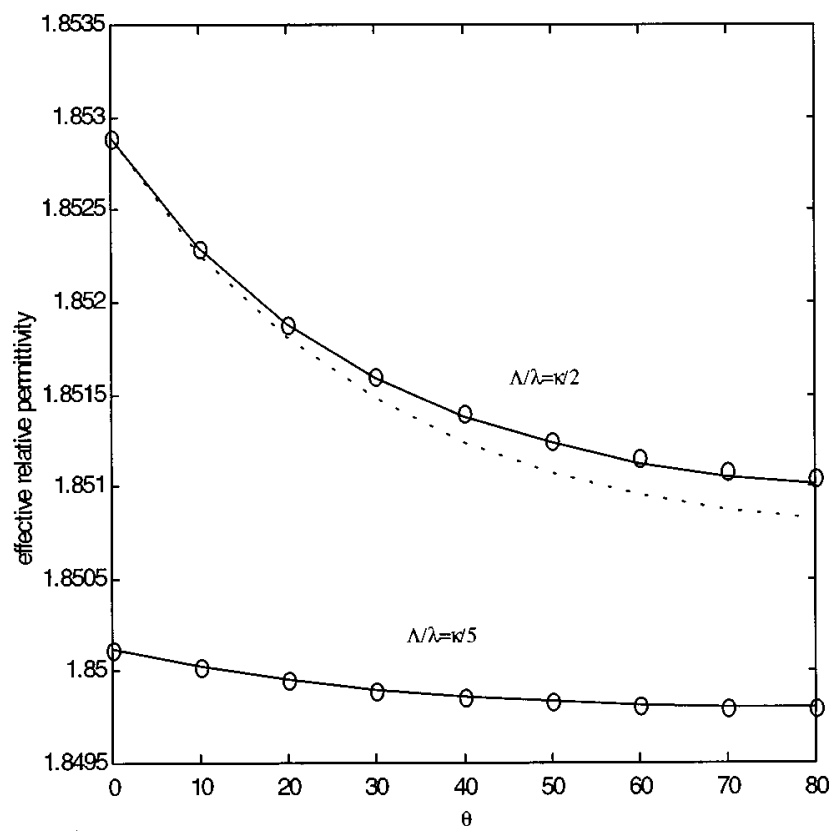

Fig. 4. Effective relative permittivity as a function of the angle of incidence for TE polarization and for different period-towavelength ratios: comparison between EMT predictions (dotted and solid curves) and results (circles) obtained by minimizing the error function of Eq. (19) by use of RCWA. Dotted and solid curves are obtained with second-order and fourth-order EMT of Eq. (17), respectively.

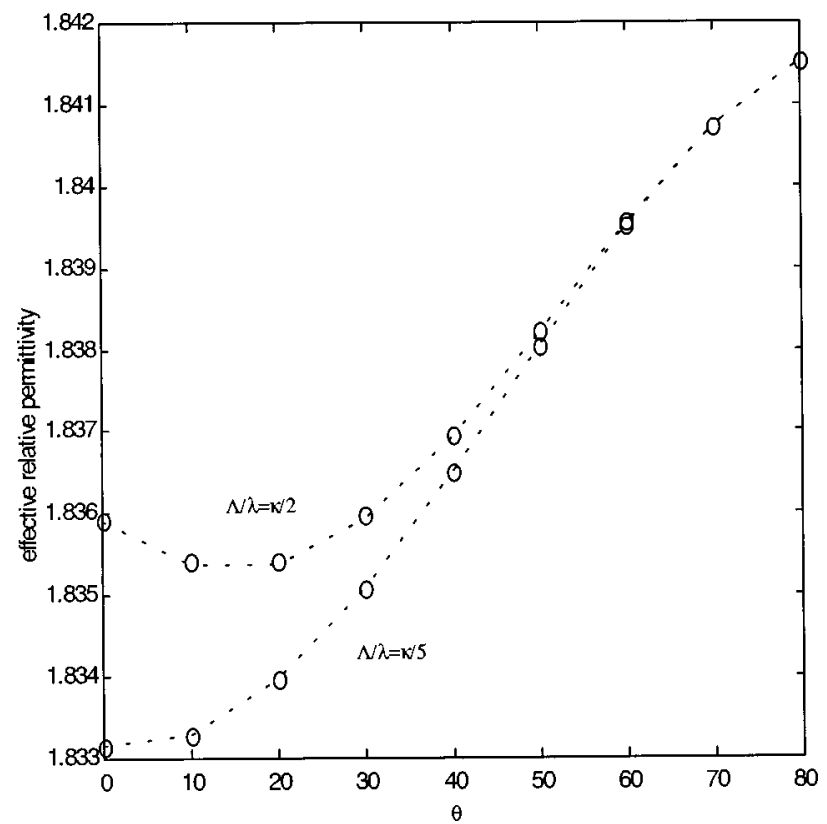

Fig. 5. Effective relative permittivity as a function of the angle of incidence for TM polarization and for different period-towavelength ratios: comparison between second-order EMT predictions (dotted curves) and results (circles) obtained by minimizing the error function of Eq. (19) by use of RCWA.
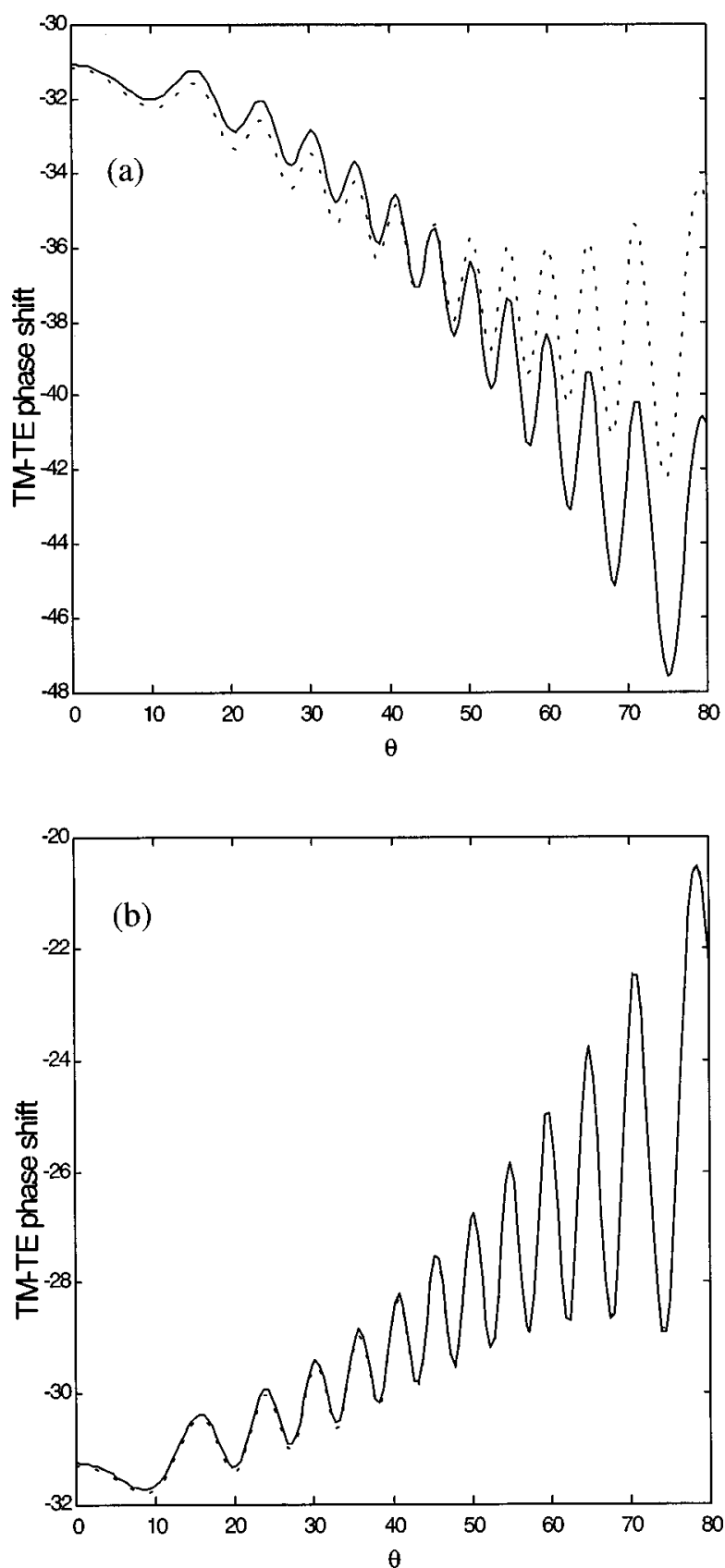

Fig. 6. TM-TE phase shift predicted by EMT (dotted curves) and RCWA (solid curves) for the dichromated gelatin grating. (a) and (b) are obtained for $\Lambda=(1 / 2)(\lambda / 1.36)$ and $(1 / 4)(\lambda / 1.36)$, respectively. They can be directly compared with those obtained with a zero-order EMT by Campbell and Kostuk [see Figs. 4(a) and 4(b) in Ref. 20].

where $r^{\prime}\left(n_{\mathrm{RCWA}}\right)$ and $t^{\prime}\left(n_{\mathrm{RCWA}}\right)$ are the reflection and the transmission complex coefficients of an 8.8- $\mu \mathrm{m}$-thick homogeneous layer with an optical index $n_{\text {RCWA }}$. A similar procedure was used in Ref. 18 to test subwavelength lamellar gratings. The value of $e$ is primordial, since it provides a quantitative answer to question (1). The answer to question (2) relies on the comparison between $n_{\mathrm{RCWA}}$ and the EMT prediction of Eqs. (17) and (18).

The values of $n_{\text {RCWA }}$ found for TE and TM polarizations are represented as circles in Figs. 4 and 5, respectively. For TE polarization, the maximum values of the error 
function obtained for $\Lambda / \lambda=\kappa / 2$ and $\kappa / 5$ are $5.9 \times 10^{-4}$ and $3.9 \times 10^{-5}$, respectively. For TM polarization, the maximum values are slightly larger and are equal to $4.8 \times 10^{-3}$ and $4.4 \times 10^{-3}$, respectively. These values are very small, and we conclude that, with good accuracy, the subwavelength volume hologram can be seen as a homogeneous thin film. For the sake of comparison, let us recall that error values of $\sim 6 \times 10^{-2}$ were observed for high-index-modulation subwavelength gratings in Ref. 18. The fact that we obtain small values for the error function $e$ is not surprising, since, in general, the equivalence between subwavelength gratings and homogeneous thin films improves when the index modulation decreases and the grating depth increases (evanescent waves can be neglected ${ }^{12}$ ). Moreover, in Figs. 4 and 5, the second- and fourth-order EMT predictions are plotted. An excellent agreement is obtained between rigorous computational results and EMT predictions; deviations between $n_{\text {RCWA }}$ and the second- and fourth-order EMT predictions are nearly invisible for TM and TE polarizations, respectively.
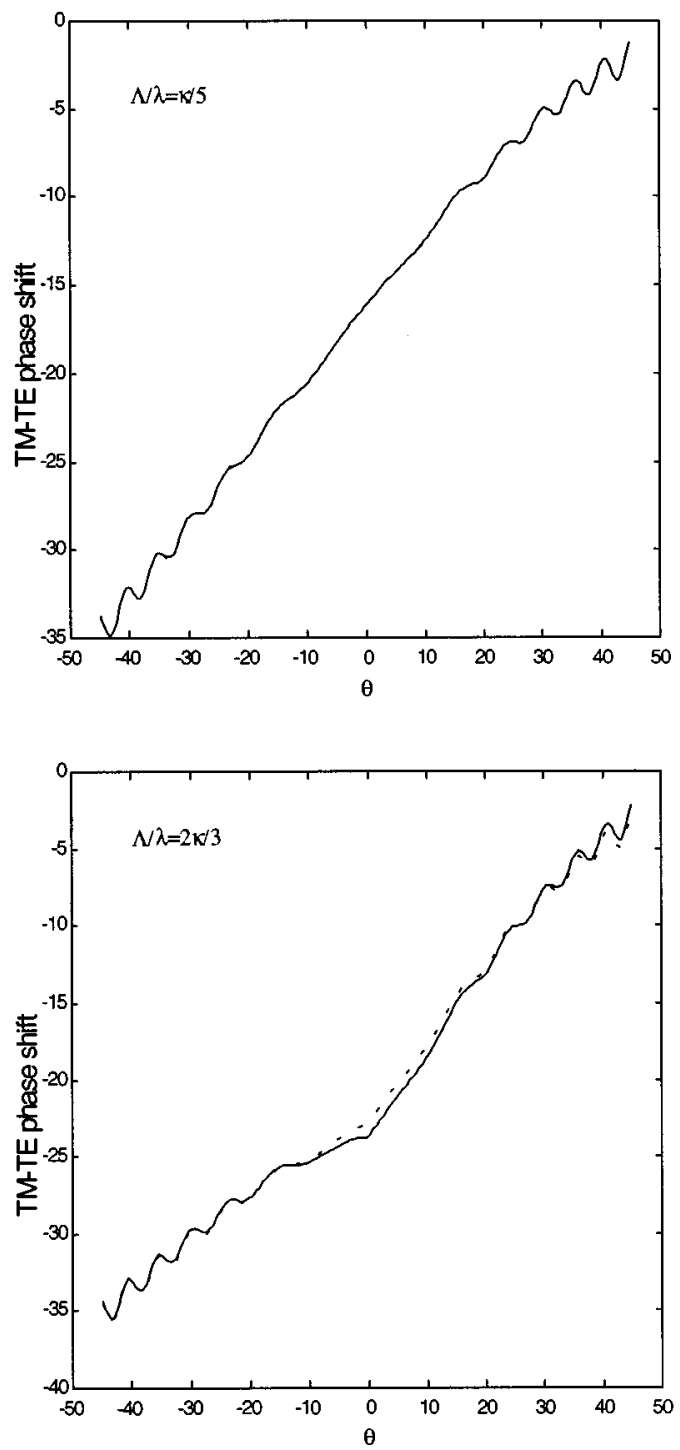

Figure 6 shows the phase difference between the TM and the TE polarizations of the zeroth-order transmitted beam as a function of the angle of incidence for two different grating periods. The solid curves show the phase difference computed by the RCWA. ${ }^{21}$ The dotted curves show the phase shifts of the equivalent birefringent thin film determined by EMT. We performed the thin-film computation with the Airy formula for homogeneous thin films, ${ }^{22}$ assuming the fourth-order approximation for TE and the second-order approximation for TM. Figure 6(b) is obtained for a volume grating with a period equal to $(1 / 4)(\lambda / 1.36) ; \lambda / 1.36$ is the wavelength in the volume hologram. As can be seen in this figure, the phase differences computed with RCWA and the birefringent thinfilm model are approximately equal; the maximum difference is smaller than $0.1^{\circ}$. At a period equal to $(1 / 2)(\lambda / 1.36)$ [Fig. 6(a)], a deviation between RCWA and thin-film computational results is observed. For angles of incidence from $0^{\circ}$ to $50^{\circ}$, the deviation does not exceed $0.7^{\circ}$. However, for larger angles of incidence the deviation is rather large, reaching $6.7^{\circ}$ for $\theta$ equal to $80^{\circ}$. Fig-
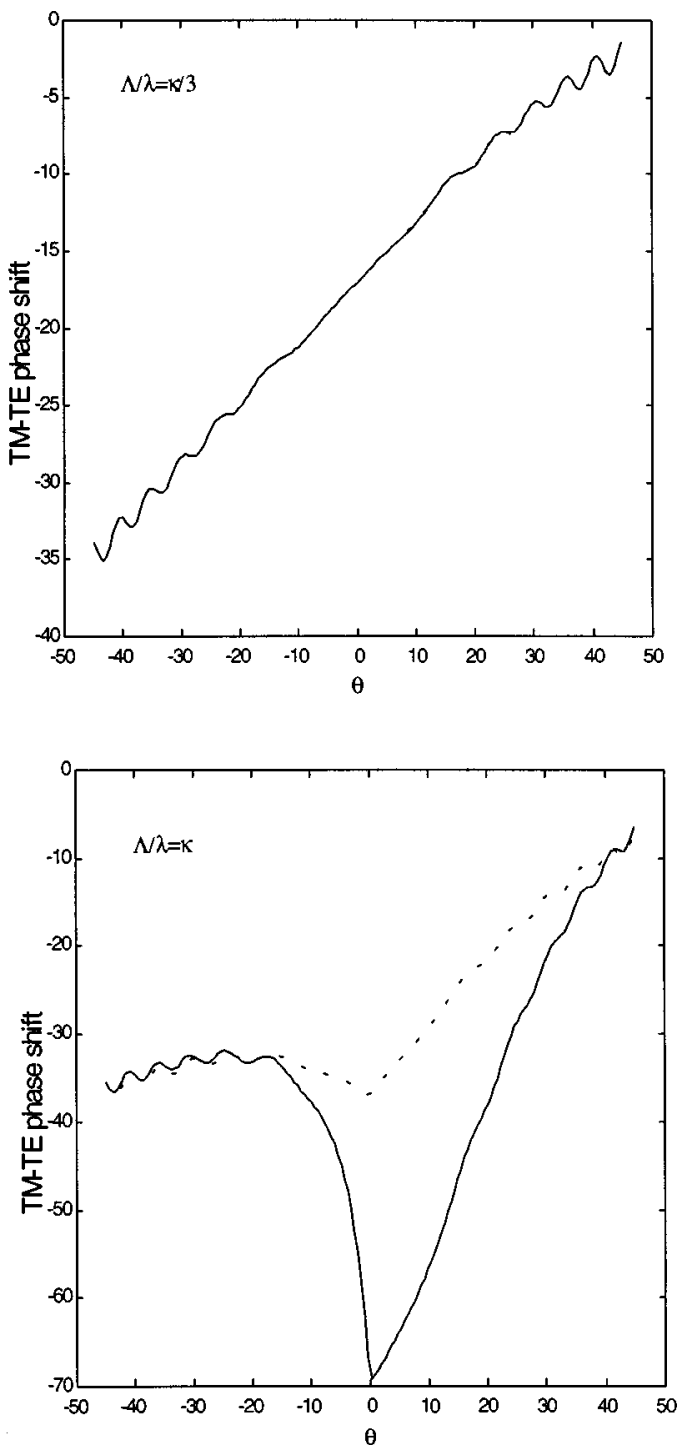

Fig. 7. TM-TE phase shift predicted by EMT (dotted curves) and RCWA (solid curves) for a slanted-fringe dichromated gelatin grating for several period-to-wavelengths, $\Lambda / \lambda=\kappa / 5, \kappa / 3,2 \kappa / 3$, and $\kappa$. The slant angle is $\Phi=45^{\circ}$. 
ures 6(a) and 6(b) can be compared directly with those obtained with a zeroth-order EMT by Campbell and Kostuk [see Figs. 4(a) and 4(b) in Ref. 19]. From this comparison, we notice that the second- and fourth-order EMT models developed in this paper drastically improve the accuracy of the birefringence thin-film computation.

\section{SLANTED VOLUME GRATINGS}

In this section we consider planar slanted-fringe grating geometries. We denote by $\Phi$ the grating slant angle and by $\left(x_{1}, z_{1}\right)$ the natural coordinate system of the grating. We have $x_{1}=x \sin \Phi+z \cos \Phi$ and $z_{1}=-x \cos \Phi$ $+z \sin \Phi$. The relative permittivity of the grating, which depends on the $x$-and $z$-coordinates, is assumed to be given by $\epsilon(x / \Lambda, z / \Lambda)=\epsilon_{0}+\Delta \epsilon \cos \left(2 \pi x_{1} / \Lambda\right)$. For slanted-fringe gratings the cutoff $\kappa$ is given by $\kappa$ $=\sin (\Phi) /\left[\max \left(n_{3}, n_{1}\right)+\beta\right]$, which reduces to $\kappa$ $=\sin (\Phi) /\left[n_{3}+\sin (\theta)\right]$ for the volume grating considered in this paper. As above, we look for a plane wave with an $x$ - and $z$-coordinate dependence equal to $\exp [j k(\gamma z$ $+\beta x)$ ], with $\beta$ given by Eq. (13). In the natural coordinate system of the grating $\left(x_{1}, z_{1}\right)$, the plane-wave expression is simply given by $\exp j k\left[(\gamma \cos \Phi+\beta \sin \Phi) x_{1}\right.$ $\left.+(\gamma \sin \Phi-\beta \cos \Phi) z_{1}\right]$, and according to the EMT of Section 2 we have

$$
(\gamma \sin \Phi-\beta \cos \Phi)^{2}=f(\gamma \cos \Phi+\beta \sin \Phi),
$$

where $f$ is the function defined by Eq. (15). In general, Eq. (20) has two solutions for $\gamma$, denoted by $\gamma_{1}$ and $\gamma_{2}$. Analytical expressions for $\gamma_{1}$ and $\gamma_{2}$ can be found by looking for a solution of Eq. (20) in a power series of $\Lambda / \lambda$. Simple expressions are obtained for TE polarization. Figure 7 shows a comparison between EMT prediction and rigorous computational results for the dichromated gelatin grating and a slant angle of $45^{\circ}$. As above, the EMT results include terms up to the fourth order of $\Lambda / \lambda$ for TE and up to the second order for TM. For the EMT computation, standard thin-film analysis software cannot be used, since the grating is not equivalent to a uniaxial layer. However, the computation is very similar and is easily handled when we assume that the electromagnetic field $U$ in the grating is a superposition of two plane waves, $U=u_{1} \exp \left[j k\left(\beta x+\gamma_{1} z\right)\right]+u_{2} \exp [j k(\beta x$ $\left.+\gamma_{2} z\right)$ ], which propagate in two homogeneous media with different effective indices, $\left(\beta^{2}+\gamma_{1}{ }^{2}\right)^{1 / 2}$ and $\left(\beta^{2}\right.$ $\left.+\gamma_{2}{ }^{2}\right)^{1 / 2}$, respectively. In Fig. 7 dashed and solid curves are obtained with the EMT and the RCWA, respectively. As can be seen, the phase differences computed with the two approaches are in excellent agreement for any angle of incidence and for $\Lambda / \lambda=\kappa / 5$ and $\kappa / 3$. For $\Lambda / \lambda=2 \kappa / 3$, a slight difference is observed. It does not exceed $1^{\circ}$. However, at cutoff a large deviation exists and the EMT is not valid.

\section{CONCLUSION}

Analytical expressions for high-order terms in $\Lambda / \lambda$ of the effective indices of arbitrary 1-D gratings were derived for slanted and unslanted geometries in classical mounting. These analytical expressions include terms up to the second- and fourth-order of $\Lambda / \lambda$, respectively, for TM and
TE polarizations. They can be applied to any periodic structure, symmetric or not, with continuously varying index profiles or step-index profiles. They are a generalization to arbitrary profiles of previous results available for lamellar gratings with one groove per period, a structure often called two-component layered media. This generalization includes the practically important case of volume holograms. It was shown that for TE polarization the normal surface of periodic structures is a sphere up to the second order only. Deviations from the sphere are found in the fourth-order term of the period-towavelength ratio. For TM polarization it was shown that the normal surface is no longer an ellipsoid of revolution at the second-order approximation. The predictions of the EMT model were compared for sinusoidally modulated volume holograms with exact electromagnetic theories to determine the limits of validity of the EMT prediction. For the grating simulated in this study (index modulation of $\sim 0.09$ ), it was found that the analogy with birefringent homogeneous media is accurate up to periodto-wavelength ratios equal to half the cutoff value for slanted and unslanted grating geometries and for any angle of incidence.

\section{ACKNOWLEDGMENTS}

We thank Céline Joubert and Brigitte Loiseaux from Laboratoire Central de Recherche, Thomson-CSF in Orsay, who initiated this study. Furthermore, we are grateful to Pierre Chavel for his comments and careful proofreading.

\section{REFERENCES}

1. S. J. Wilson and M. C. Hutley, "The optical properties of 'moth eye' antireflection surfaces," Opt. Acta 29, 993-1009 (1982).

2. R. C. Enger and S. K. Case, "Optical elements with ultrahigh spatial-frequency surface corrugations," Appl. Opt. 22, 3220-3228 (1983).

3. T. K. Gaylord, W. E. Baird, and M. G. Moharam, "Zeroreflectivity high spatial-frequency rectangular-groove dielectric surface-relief gratings," Appl. Opt. 25, 4562-4567 (1986).

4. Y. Ono, Y. Kimura, Y. Otha, and N. Nishida, "Antireflection effects in ultrahigh spatial-frequency holographic relief gratings," Appl. Opt. 26, 1142-1146 (1987).

5. M. E. Motamedi, W. H. Southwell, and W. J. Gunning, "Antireflection surfaces in silicon using binary optics technology," Appl. Opt. 31, 4371-4376 (1992).

6. D. H. Raguin and G. M. Morris, "Antireflection structured surfaces for the infrared spectral region," Appl. Opt. 32, 1154-1167 (1993).

7. L. H. Cescato, E. Gluch, and N. Streibl, "Holographic quarterwave plates," Appl. Opt. 29, 3286-3290 (1990).

8. D. C. Flanders, "Submicrometer periodicity gratings as artificial anisotropic dielectrics," Appl. Phys. Lett. 42, 492494 (1983).

9. P. Yeh, "A new optical model for wire grid polarizers," Opt. Commun. 26, 289-292 (1978).

10. W. Stork, N. Streibl, H. Haidner, and P. Kipfer, "Artificial distributed-index media fabricated by zero-order gratings," Opt. Lett. 16, 1921-1923 (1991).

11. F. T. Chen and H. G. Craighead, "Diffractive phase elements based on two-dimensional artificial dielectrics," Opt. Lett. 20, 121-123 (1995).

12. Ph. Lalanne and D. Lemercier-Lalanne, "Depth dependence of the effective properties of subwavelength gratings," J. Opt. Soc. Am. A 14, 450-458 (1997). 
13. G. Bouchitté and R. Petit, "Homogenization techniques as applied in the electromagnetic theory of gratings," Electromagnetics 5, 17-36 (1985).

14. R. C. McPhedran, L. C. Botten, M. S. Craig, N. Nevière, and D. Maystre, "Lossy lamellar gratings in the quasi-static limit," Opt. Acta 29, 289-312 (1982).

15. S. M. Rytov, "Electromagnetic properties of a finely stratified medium," Sov. Phys. JETP 2, 466-475 (1956).

16. C. Gu and P. Yeh, "Form birefringence dispersion in periodic layered media," Opt. Lett. 21, 504-506 (1996).

17. J. M. Bell, G. H. Derrick, and R. C. McPhedran, "Diffraction gratings in the quasi-static limit," Opt. Acta 29, 1475-1489 (1982).
18. Ph. Lalanne and D. Lemercier-Lalanne, "On the effective medium theory of subwavelength periodic structures," J. Mod. Opt. 43, 2063-2085 (1996).

19. G. Campbell and R. K. Kostuk, "Effective-medium theory of sinusoidally modulated volume holograms," J. Opt. Soc. Am. A 12, 1113-1117 (1995).

20. P. Yeh, Optical Waves in Layered Media (Wiley, New York, 1988), Chap. 6.

21. T. K. Gaylord and M. G. Moharam, "Analysis and application of optical diffraction by grating," Proc. IEEE 73, 894936 (1985).

22. M. Born and E. Wolf, Principles of Optics, 6th ed. (Pergamon, New York, 1984), p. 62. 